\title{
Estudo comparativo do perfil de idosos atendidos em um hospital terciário: ambulatório de psiquiatria geriátrica e unidade de internação psiquiátrica
}

\author{
Comparative study of the profile of elderly served in a tertiary hospital: \\ ambulatory of geriatric psychiatry and unit of psychiatric intervention
}

\section{Estudio comparativo del perfil de idosos atendidos en un hospital terciario: ambulatorio de psiquiatría geriátrica y unidad de internation psiquiatrica}

\author{
Letícia Güenter ${ }^{\mathbb{D}}$ 1, Gabriel Behr Gomes Jardim (iD) 1, Francisco Pascoal Jr (D) 1, \\ Lucas Spanemberg ${ }^{(1}$ ', Marco Antônio Pacheco ${ }^{1}$ ', Vanessa Sgnaolin ${ }^{\circledR}{ }^{1} \bowtie$, Alfredo Cataldo Neto ${ }^{1}$ \\ Pontifícia Universidade Católica do Rio Grande do Sul (PUCRS). Porto Alegre, RS, Brasil.
}

\section{ARTICLE INFO}

\section{Article history}

Received: 03/05/2019

Accepted: 27/06/2019

Published: 29/08/2019

$\triangle$ Correspondent Author

Vanessa Sgnaolin

Av. Ipiranga, 6681 - Prédio 81 sala 703

90619-900, Porto Alegre, RS, Brasil

vanessa.sgnaolin@pucrs.br

\section{(C) 2019 All rights reserved}

\section{Editors}

Alfredo Cataldo Neto

Newton Luiz Terra

Assistant Editors

Paula Engroff

Vanessa Sgnaolin

\begin{abstract}
RESUMO
OBJETIVOS: Verificar o perfil de idosos e seus principais diagnósticos no ambulatório de psiquiatria geriátrica e unidade de internação psiquiátrica de um hospital terciário.

MÉTODOS: Estudo transversal de base populacional realizado em um hospital terciário de referência, com indivíduos com idade $\geq 55$ anos atendidos no ambulatório de psiquiatria geriátrica e na internação psiquiátrica, no período de 2014 a 2018. Os dados foram coletados por meio de entrevista. As variáveis estudadas foram sociodemográficas e aspectos clínicos.

RESULTADOS: Foram avaliados 497 indivíduos, destes 269 procedentes do ambulatório

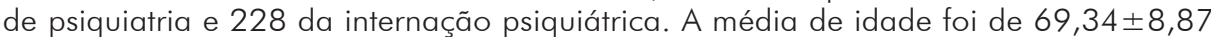
anos. A maioria dos pacientes eram do sexo feminino (75,8\%), não possuíam companheiro $(56,3 \%)$, tinham o ensino fundamental $(50,3 \%)$, residiam em Porto Alegre $(75,6 \%)$ e eram aposentados $(66,5 \%)$. De acordo com as variáveis clínicas estudadas, $89,6 \%$ dos indivíduos possuíam alguma morbidade, 36,9\% já haviam sido internados em ala psiquiátrica, o diagnóstico mais encontrado foi de depressão $(40,8 \%)$, seguido de transtorno do humor bipolar (19\%) e ansiedade (14,1\%).

CONCLUSÕES: Com o envelhecimento populacional é esperado o aumento das morbidades e dos transtornos mentais, exigindo ferramentas diagnósticas mais específicas e melhores abordagens de tratamento. Ainda não existem dados consistentes sobre o manejo dos transtornos do humor nos idosos, sendo assim, nosso estudo pode nortear um melhor atendimento aos idosos e indivíduos que estão envelhecendo, aprimorando o planejamento dos serviços e políticas de saúde.
\end{abstract}

DESCRITORES: Envelhecimento; Psiquiatria; Depressão; Ansiedade.

\footnotetext{
ABSTRACT

AIMS: To verify the profile of the elderly and their main diagnoses in the geriatrics psychiatric outpatient clinic and the psychiatric hospitalization unit of a tertiary hospital.

METHODS: A cross-sectional study of population-based was performed in a tertiary referral hospital, with individuals aged $\geq 55$ years attending the geriatric psychiatric outpatient clinic and psychiatric hospitalization, from 2014 to 2018 . Data were collected through interviews. The studied variables were sociodemographic and clinical aspects.
} 
RESULTS: A total of 497 individuals were evaluated, 269 from the psychiatric outpatient clinic and 228 from the psychiatric hospital. The mean age was $69.34 \pm 8.87$ years. Most of the patients were female (75.8\%), had no partner (56.3\%), had primary education (50.3\%), lived in Porto Alegre (75.6\%) and were retired (66.5\%). According to the clinical variables studied, $89.6 \%$ of the individuals had some morbidity, $36.9 \%$ had already been admitted to the psychiatric ward, the most common diagnosis was depression (40.8\%), followed by bipolar disorder (19\%) and anxiety (14.1\%).

CONCLUSIONS: With the population aging, it is expected to increase morbidity and mental disorders, requiring more specific diagnostic tools and better treatment approaches. There are still no consistent data on the management of mood disorders in the elderly, so our study can guide a better care for elderly and aging individuals, improving the planning of health services and policies.

KEYWORDS: Aging; Psychiatry; Depression; Anxiety.

\section{RESUMEN}

OBJETIVOS: Verificar el perfil de ancianos y sus principales diagnósticos en el ambulatorio de psiquiatría geriátrica y unidad de internación psiquiátrica de un hospital terciario.

MÉTODOS: Estudio transversal de base poblacional realizado en un hospital terciario de referencia, con individuos con edad $\geq 55$ años atendidos en el ambulatorio de psiquiatría geriátrica y en la internación psiquiátrica, en el período de 2014 a 2018 . Los datos fueron recolectados por medio de entrevista. Las variables estudiadas fueron sociodemográficas y aspectos clínicos.

RESULTADOS: Se evaluaron 497 individuos, de estos 269 procedentes del ambulatorio de psiquiatría y 228 de la internación psiquiátrica. El promedio de edad fue de 69,34 4 8,87 años. La mayoría de los pacientes eran del sexo femenino (75,8\%), no tenían compañero (56,3\%), tenían la enseñanza fundamental (50,3\%), residían en Porto Alegre $(75,6 \%)$ y eran jubilados $66,5 \%)$. De acuerdo con las variables clínicas estudiadas, el $89,6 \%$ de los individuos poseía alguna morbilidad, el 36,9\% ya habían sido internados en ala psiquiátrica, el diagnóstico más encontrado fue de depresión (40,8\%), seguido de trastorno del humor bipolar (19\%) y ansiedad (14,1\%).

CONCLUSIONES: Con el envejecimiento poblacional se espera el aumento de las morbilidades y de los trastornos mentales, exigiendo herramientas diagnósticas más específicas y mejores abordajes de tratamiento. No hay datos consistentes sobre el manejo de los trastornos del humor en los ancianos, por lo que nuestro estudio puede guiar una mejor atención a los ancianos e individuos que están envejeciendo, mejorando la planificación de los servicios y políticas de salud.

Palabras clave: Envejecimiento; Psiquiatría; Depresión; Ansiedad.

\section{INTRODUÇÃO}

Com o aumento da expectativa de vida, é notável o envelhecimento populacional. Esta mudança ocorreu antecipadamente nos países desenvolvidos e, recentemente, nos países emergentes - como o Brasil - devido a diversos fatores, como diminuição na taxa de fecundidade, melhorias de saúde e socioeconômica. ${ }^{1}$

Em todo o mundo, espera-se que fração de indivíduos com mais de 60 cresça 56\% entre 2015 e 2030. Com a mudança demográfica se alterou o perfil de saúde, onde $23 \%$ da carga global total de doenças é agora atribuível aos idosos. ${ }^{2}$ Esta mudança está ligada a desafios econômicos, culturais, médicos, sociais, de saúde pública e de políticas públicas. ${ }^{3}$

A multimorbidade, definida como a presença de mais de duas comorbidades clínicas em uma mesma pessoa, é um problema de saúde pública e mundial, e está associada a desfechos negativos, principalmente devido a sua alta prevalência. , $^{5}$ Até $24,2 \%$ dos adultos brasileiros possuem multimorbidade de condições crônicas, podendo variar amplamente dependendo do perfil de cada indivíduo. ${ }^{6}$

Estima-se que nos próximos 20 anos haverá uma expansão da morbidade, principalmente a multimorbidade, sendo a patologia mental uma das principais, podendo representar dois terços dos casos. ${ }^{7}$ Os transtornos do humor representam a fonte mais comum de morbidade psiquiátrica em idosos, ${ }^{8}$ sendo a depressão, ansiedade e o transtorno do humor bipolar os mais prevalentes com graus variados de gravidade. ${ }^{9-11}$

Desta forma, torna-se imprescindível avaliarmos e entendermos o perfil dos indivíduos de meia-idade e idosos, bem como seus principais diagnósticos, visto que a tendência é aumentar a demanda de atendimento desta população nos ambulatórios e na internação psiquiátrica hospitalar.

\section{MÉTODOS}

Trata-se de um estudo transversal com base populacional realizado no Hospital São Lucas da PUCRS, um dos hospitais terciários de referência do município de Porto Alegre-RS. O estudo abrangeu todos os indivíduos com idade igual ou superior a 55 anos que foram atendidos no ambulatório de psiquiatria geriátrica e na internação psiquiátrica no período de 2014 a 2018. Entre os pacientes com mais de uma admissão, apenas a última foi incluída.

Os dados foram coletados em atendimento médico, seguindo um questionário geral pré-projetado, que eram coletados na primeira consulta para os pacientes do ambulatório e na admissão para os provenientes da internação.

As informações sociodemográficas obtidas nos questionários geral foram idade, gênero, estado civil, 
nível de escolaridade, procedência, situação ocupacional (aposentado ou não). Os aspectos clínicos avaliados foram a presença de alguma patologia, internação psiquiátrica prévia e diagnóstico estabelecido no final da consulta/internação pelos médicos que realizaram o atendimento, com base no DSM 5 .

\section{Aspectos éticos}

O presente trabalho foi aprovado pelo Comitê de Ética em Pesquisa da Pontifícia Universidade Católica do Rio Grande do Sul sob o parecer 1072.823.737 e pela Comissão Científica do Instituto de Geriatria e Gerontologia da PUCRS (SIPESQ: 8322).

\section{Análise estatística}

Os dados analisados no programa SPSS (do inglês, Statistical Package for the Social Sciences) versão 19. As variáveis foram descritas por frequências, médias e desvios padrões. Para verificar a significância, as variáveis quantitativas foram testadas com o teste t, e para as variáveis categóricas, foram testadas através do teste do qui-quadrado de Pearson.

\section{RESULTADOS}

\section{Perfil sociodemográfico}

A amostra foi composta de 497 indivíduos com 55 anos ou mais, dos quais 269 dos indivíduos eram procedentes do ambulatório de psiquiatria e 228 da internação psiquiátrica. A média de idade da população estudada foi de 69,34 anos (DP 8,87). No grupo dos pacientes que receberam tratamento ambulatorial a média de idade foi de 72,29 anos (DP 8,46) e no grupo proveniente da internação a média foi de 65,86 anos (DP 8,07).

A Tabela 1 demostra as características sociodemográficas da população estudada. Entre os pacientes que receberam tratamento ambulatorial $75,8 \%$ eram do sexo feminino. Porém, entre os pacientes internados $53,1 \%$ eram do sexo feminino e $46,9 \%$ do sexo masculino.

Quanto ao estado civil, foi verificado que 56,3\% dos indivíduos atendidos não possuíam companheiro. Ao dividirmos as categorias $45,8 \%$ eram casados ou viviam com companheiro, $8,9 \%$ eram solteiros, $17,7 \%$ separados e $27,6 \%$ viúvos. No grupo proveniente do ambulatório, quando avaliado os indivíduos que não possuíam companheiro a maioria eram viúvos $(34,8 \%)$. Por outro lado, no grupo proveniente da internação, 23,1\% estavam separados.

A respeito da escolaridade, boa parte dos idosos apresentavam baixo nível de escolaridade, sendo $2,3 \%$ analfabetos, $50,3 \%$ ensino fundamental, $27,8 \%$ ensino médio, $15 \%$ ensino superior e $4,6 \%$ eram pós-graduados. A amostra foi equivalente para ambos os grupos.

Tabela 1. Características sociodemográficas da população estudada.

\begin{tabular}{|c|c|c|c|c|}
\hline & $\begin{array}{c}\text { Total } \\
\mathbf{n}=497\end{array}$ & $\begin{array}{c}\text { Ambulatório } \\
\mathbf{n}=269\end{array}$ & $\begin{array}{c}\text { Internação } \\
\mathbf{n}=\mathbf{2 2 8}\end{array}$ & $\mathbf{p}$ \\
\hline Idade, média (DP) & $69,34(8,87)$ & $72,29(8,46)$ & $65,86(8,07)$ &, 000 \\
\hline \multicolumn{5}{|l|}{ Gênero, $n(\%)$} \\
\hline Feminino & $325(65,4)$ & $204(75,8)$ & $121(53,1 \%)$ & \multirow{2}{*}{, 000} \\
\hline Masculino & $172(34,6)$ & $65(24,2)$ & $107(46,9 \%)$ & \\
\hline \multicolumn{5}{|l|}{ Estado Civil, $n(\%)$} \\
\hline Possui companheiro(a) & $217(45,8)$ & $111(43,9)$ & $106(48,0)$ & \multirow{4}{*}{, 001} \\
\hline Solteiro (a) & $42(8,9)$ & $21(8,3)$ & $21(9,5)$ & \\
\hline Divorciado (a) & $84(17,7)$ & $33(13,0)$ & $51(23,1)$ & \\
\hline Viúvo (a) & $131(27,6)$ & $88(34,8)$ & $43(19,5)$ & \\
\hline \multicolumn{5}{|l|}{ Escolaridade, $n(\%)$} \\
\hline Analfabeto & $11(2,3)$ & $5(1,9)$ & $6(2,8)$ & \multirow{5}{*}{,009 } \\
\hline Fundamental & $241(50,3)$ & $145(55,6)$ & $96(44,0)$ & \\
\hline Ensino Médio & $133(27,8)$ & $74(28,4)$ & $59(27,1)$ & \\
\hline Superior & $72(15,0)$ & $26(10,0)$ & $46(21,1)$ & \\
\hline Pós-graduação & $22(4,6)$ & $11(4,2)$ & $11(5,0)$ & \\
\hline \multicolumn{5}{|l|}{ Procedência, $n(\%)$} \\
\hline Capital RS & $362(75,6)$ & $170(66,7)$ & $192(85,7)$ & \multirow{3}{*}{, 000} \\
\hline Outros RS & $115(24,0)$ & $84(32,9)$ & $31(13,8)$ & \\
\hline Outros & $2(0,4)$ & $1(0,4)$ & $1(0,4)$ & \\
\hline Aposentado, $n(\%)$ & $323(66,5)$ & $208(79,1)$ & $115(51,6)$ & 000 \\
\hline
\end{tabular}


Três quartos dos idosos residiam em Porto Alegre (75,6\%), 24,0\% eram procedentes de outras cidades do Rio Grande do Sul e 0,4\% de outros locais. Ao observar o grupo proveniente da internação foi verificado que cerca de 85,7\% eram procedentes de Porto Alegre, sendo significativamente superior ao ambulatório onde $66,7 \%$ eram procedentes da capital.

A maioria dos idosos do nosso grupo eram aposentados $(66,5 \%)$, sendo que o grupo proveniente do ambulatório $(79,1 \%)$ foi significativamente superior ao da internação $(51,6 \%)$.

\section{Perfil clínico}

A respeito das comorbidades clínicas, $89,6 \%$ dos indivíduos possuíam alguma morbidade, sendo esta variável correspondente entre os grupos de ambulatório e internação. Cerca de $36,9 \%$ dos indivíduos atendidos já haviam sido internados em ala psiquiátrica, sendo a maior incidência no grupo proveniente da internação $(58,3 \%)$.

Como observado na Tabela 2 o diagnóstico mais encontrado foi de depressão, representando 40,8\% dos casos, seguido de transtorno do humor bipolar (19\%) e ansiedade $(14,1 \%)$. Tanto no grupo do ambulatório quanto no grupo da internação, o diagnóstico de depressão se mostrou o mais prevalente, porém, este diagnóstico foi significativamente maior no grupo proveniente do ambulatório (44,6\%) quando comparado com a internação (36,4\%). Encontramos como segundo diagnóstico mais prevalente no ambulatório os transtornos de ansiedade, representando 24,8\% dos casos e sendo significativamente superior ao grupo da internação com apenas 1,8\%. Em contrapartida, o grupo proveniente da internação apresentou como seu segundo diagnóstico mais prevalente o transtorno de humor bipolar (32\%), sendo este grupo significativamente superior ao ambulatório $(7,8 \%)$.

\section{DISCUSSÃO}

Com o aumento progressivo do envelhecimento populacional, observa-se um aumento na incidência de transtornos psiquiátricos na faixa etária idosa. Assim sendo, estudos que abrangem o perfil sociodemográfico dessa faixa etária têm ganho destaque. A média de idade do grupo de pacientes que receberam tratamento ambulatorial foi significativamente maior $(72,29 \pm 8,46$ anos), quando comparada com grupo proveniente da internação $(65,86 \pm 8,07$ anos) .

Sabemos que em praticamente todos os transtornos mentais o sexo feminino é mais prevalentemente diagnosticado. ${ }^{8,12,13}$ Neste estudo, apesar do sexo feminino ser o mais prevalente em ambos os grupos, nota-se um aumento significativo naqueles pacientes provenientes do ambulatório. Ao observar os idosos do sexo masculino, verificou-se que estes procuravam a internação com muito mais frequência que o ambulatório. Os pacientes do sexo masculino costumam ter menor aderência ao tratamento, podendo internar com mais frequência. ${ }^{14}$ Entretanto, a avaliação de gênero no grupo de pacientes provenientes da internação pode ter sido prejudicada, em virtude dos leitos da unidade de internação psiquiátrica comportarem obrigatoriamente $50 \%$ do sexo feminino e $50 \%$ do sexo masculino.

Foi demonstrado que $51,8 \%$ dos idosos não possuíam companheiro e este fator está associado a uma piora na saúde mental. ${ }^{15,16} \mathrm{O}$ fato de possuir companheiro está associado à redução da solidão e melhoria do bemestar. ${ }^{17} \mathrm{O}$ grupo advindo do ambulatório apresentou um número significativo de viúvos (38\%). O luto

Tabela 2. Aspectos clínicos-patológicos da população estudada.

\begin{tabular}{|c|c|c|c|c|}
\hline & $\begin{array}{c}\text { Total } \\
n=497\end{array}$ & $\begin{array}{c}\text { Ambulatório } \\
\mathbf{n}=269\end{array}$ & $\begin{array}{c}\text { Internação } \\
\mathbf{n}=\mathbf{2 2 8}\end{array}$ & $\mathbf{p}$ \\
\hline Comorbidade, $n(\%)$ & $439(89,6)$ & $233(88,6)$ & $206(90,7)$ & ,265 \\
\hline Int. Psiquiátrica, $n(\%)$ & $178(36,9)$ & $51(19,2)$ & $127(58,3)$ & 000 \\
\hline \multicolumn{5}{|l|}{ Diagnóstico, $n(\%)$} \\
\hline Depressão & $197(40,8)$ & $115(44,6)$ & $82(36,4)$ & 000 \\
\hline Ansiedade & $68(14,1)$ & $64(24,8)$ & $4(1,8)$ & 000 \\
\hline Depressão/Ansiedade & $6(1,2)$ & $6(2,3)$ & $0(0,0)$ &, 000 \\
\hline Transtorno H. Bipolar & $92(19,0)$ & $20(7,8)$ & $72(32,0)$ &, 000 \\
\hline Transtorno Personalidade, $n(\%)$ & $7(1,4)$ & $3(1,2)$ & $4(1,8)$ &, 000 \\
\hline Uso de Substancias & $24(5,0)$ & $5(1,9)$ & $19(8,4)$ &, 000 \\
\hline Patologias Demências & $45(9,3)$ & $30(11,6)$ & $15(6,7)$ &, 000 \\
\hline Patologias Psicóticas & $39(8,1)$ & $10(3,9)$ & $29(12,9)$ &, 000 \\
\hline Outros & $5(1,0)$ & $5(1,9)$ & $0(0,0)$ &, 000 \\
\hline
\end{tabular}


de um ente querido resulta em isolamento social e solidão, que são fatores de risco significativos para a saúde mental e baixa qualidade de vida. ${ }^{18}$ Por outro lado, no grupo proveniente da internação foi observado 23,1\% de divorciados. Provavelmente, estes pacientes, devido ao seu próprio perfil, tinham mais dificuldade de manter relações interpessoais. $\mathrm{O}$ apoio e o convívio social refletem nos resultados e melhores condições de saúde mentais, levando a um envelhecimento bemsucedido. ${ }^{16}$

Mais da metade dos idosos apresentaram baixo nível de escolaridade, sendo esta distribuição equivalente para ambos os grupos. Diversos estudos afirmam que a presença de um baixo nível de escolaridade está relacionada com maior prevalência nos transtornos mentais. ${ }^{4,12,19}$ Sendo assim, quanto maior a reserva cognitiva, menor a possibilidade de desenvolver demência.

As evidências a respeito de como a procedência, rural ou urbana, interfere na prevalência dos transtornos de humor é controversa na literatura. Alguns autores não encontraram relação com a procedência. ${ }^{20,21}$ Por outro lado, existem estudos comprovando que o diagnóstico de depressão é mais prevalente em áreas urbanas. ${ }^{22,23}$ Em contrapartida, estudos defendem que proceder de área rural é fator de risco..$^{15,22}$ Foi verificado que $75,6 \%$ dos idosos eram procedentes de Porto Alegre, onde no grupo procedente do ambulatório a amostra correspondia a $66,7 \%$ dos pacientes, já na internação o grupo foi significativamente maior com $85,7 \%$. Entretanto, cabe salientar que a unidade de internação psiquiátrica funciona com $50 \%$ dos leitos privativos e os outros $50 \%$ de atendimento SUS, onde estes, obrigatoriamente, devem pertencer à cidade de Porto Alegre, gerando assim um aumento em sua proporção.

A probabilidade de apresentar um episódio depressivo maior é menor para idosos aposentados do que para aqueles que estão trabalhando. ${ }^{24} \mathrm{O}$ fato de estar aposentado melhora a qualidade de vida, principalmente no que diz respeito a condição socioeconômica, possibilitando, por exemplo, acesso a melhores condições de saúde. ${ }^{25}$ Cerca de dois terços do grupo de indivíduos atendidos no ambulatório eram aposentados, sendo significativamente maior do que o grupo advindo da internação. Desta forma, pode-se inferir que aqueles pacientes que não eram aposentados sofriam mais com as adversidades do ambiente, aumentando assim sua carga de estresse, o que contribui para o surgimento dos transtornos mentais.

Com o envelhecimento populacional espera-se também um aumento nas morbidades clínicas. ${ }^{7,26}$ As doenças crônicas são vistas como importantes fatores de risco para a saúde mental, pois podem reduzir a capacidade de reserva fisiológica, aumentando os níveis de estresse. ${ }^{27,28} \mathrm{~A}$ frequência de multimorbidade no Brasil foi de $22,2 \% .^{4}$ A prevalência de qualquer transtorno de saúde mental aumenta significativamente com o número de condições físicas. ${ }^{6}{ }^{29}$ Segundo os dados encontrados mais de um terço dos paciente apresentaram alguma comorbidade clínica, porém esta variável não se mostrou significativamente diferente entre os grupos ambulatorial e internação. Desta forma, a presença de comorbidade clínica parece não interferir na gravidade dos transtornos mentais, porém, devido sua grande prevalência, parece contribuir para o surgimento da mesma e vice-versa.

Aproximadamente 32\% dos pacientes geriátricos que sofrem de alguma doença mental necessitará de uma admissão psiquiátrica em um período de 5 anos..$^{14,30}$ Neste estudo cerca de 36,9\% dos pacientes já haviam sido internados em ala psiquiátrica, sendo o grupo pertencente ao ambulatório (19,2\%) significativamente menor que grupo da internação $(58,3 \%)$. Esses dados condizem com a literatura atual, a qual afirma que os pacientes que aderem ao tratamento ambulatorial apresentam-se como menos graves, diminuindo a necessidade de internação em unidade psiquiátrica. ${ }^{14,31}$

A depressão é um dos transtornos mentais mais prevalentes em todo o mundo, ${ }^{10,32}$ podendo variar de 1 a $14 \%$ para os diagnósticos de Depressão Maior e $9,8 \%$ a $54 \%$ para sintomas depressivos em geral. ${ }^{33-35} \mathrm{~A}$ depressão foi o diagnóstico mais prevalente tanto para a internação quanto para o ambulatório, correspondendo 40,8\% dos casos. O diagnóstico de depressão é um dos principais responsáveis por aumento de incapacidades, risco de morbidade e mortalidade, designando-a como uma importante prioridade de saúde pública. ${ }^{32}$

O Transtorno do Humor Bipolar (THB) foi o segundo diagnóstico mais prevalente, representando $19 \%$ dos idosos atendidos, onde 7,8\% pertenciam ao ambulatório e 32\% na internação. De todos os pacientes com THB, 25\% tinham mais de 60 anos e espera-se que esse número aumente para $50 \%$ em 2030. ${ }^{36} \mathrm{~A}$ prevalência gira em torno de $0,5 \%$ a $1,0 \%$ dos adultos com idade superior a 60 anos. ${ }^{37}$ É responsável por $6 \%$ das visitas ambulatoriais de psiquiatria geriátrica, $8-10 \%$ das internações geriátricas e 17\% dos idosos em salas de emergência psiquiátrica. ${ }^{38}$ Idosos com o diagnóstico de THB possuem mais chances de necessitar de uma internação psiquiátrica durante a sua vida e apresentam mais probabilidade de reinternações. ${ }^{14} \mathrm{~A}$ prevalência de THB foi significativamente maior do que a encontrada em outros estudos, isso se deve ao fato destes resultados já representarem uma consequência do envelhecimento 
populacional e também por nosso grupo estudar este tipo de diagnóstico.

Segundo a organização mundial da saúde, estima-se que $7,7 \%$ da população feminina sofram de transtorno de ansiedade (homens, 3,6\%)..$^{10}$ A prevalência dos transtornos de ansiedade aumentou de 2005 para 2015 cerca de 14,9\%, provavelmente decorrente do crescimento populacional e do envelhecimento. ${ }^{32}$ Estudos populacionais em idosos demonstraram uma grande variação na prevalência de ansiedade, variando de 3,1 a $30 \%$, ${ }^{39}$ porém a ansiedade é frequentemente subdiagnosticada entre pessoas mais velhas, pois muitas vezes se apresenta como uma comorbidade nos transtornos mentais. ${ }^{40} \mathrm{O}$ transtorno de ansiedade foi o segundo diagnóstico mais prevalente nos indivíduos provenientes do ambulatório $(24,8 \%)$ e o terceiro mais prevalente na amostra geral $(14,1 \%)$, correspondendo desta forma aos achados na literatura.

O presente estudo apresenta as limitações de um estudo transversal e descritivo, como a falta de controle de qualidade das informações fornecidas e de verificações cruzadas entre as variáveis, e a impossibilidade de estabelecer a temporalidade dos fatores associados. Também apresenta resultados que se aplicam a uma população delimitada e que foi incluída por conveniência.

\section{CONSIDERAÇÕES FINAIS}

Com o envelhecimento populacional é esperado o aumento das morbidades e dos transtornos mentais, exigindo assim, ferramentas diagnósticas mais específicas e melhores abordagens de tratamento. Ainda não existem dados consistentes sobre o manejo dos transtornos do humor nos idosos, principalmente devido as altas taxas de comorbidades nesta população. Sendo assim, nosso estudo pode ajudar a nortear um melhor atendimento dos idosos, aprimorando o planejamento dos serviços e políticas de saúde.

\section{REFERÊNCIAS}

1. Miranda GMD, Mendes AdCG, da Silva ALA. O envelhecimento populacional brasileiro: desafios e consequências sociais atuais e futuras. Rev Bras Geriatr Gerontol. 2016;19(3):507-19.

2. Prince MJ, Wu F, Guo Y, Robledo LMG, O'Donnell M, Sullivan R, Yusuf S. The burden of disease in older people and implications for health policy and practice. Lancet. 2015;385(9967):549-62. https://doi.org/10.1016/s0140 $6736(14) 61347-7$

3. Sander M, Oxlund B, Jespersen A, Krasnik A, Mortensen EL, Westendorp RGJ, Rasmussen LJ. The challenges of human population ageing. Age Ageing. 2014;44(2):185-7. https://doi.org/10.1093/ageing/afu189
4. Nunes BP, Chiavegatto Filho AD, Pati S, Teixeira DSC, Flores TR, Camargo-Figuera FA, Munhoz TN, Thumé E, Facchini LA, Batista SRR. Contextual and individual inequalities of multimorbidity in Brazilian adults: a crosssectional national-based study. BMJ Open. 2017;7(6): e015885. https://doi.org/10.1136/bmjopen-2017-015885

5. Nunes BP, Flores TR, Mielke GI, Thume E, Facchini LA. Multimorbidity and mortality in older adults: a systematic review and meta-analysis. Arch Gerontol Geriatr. 2016;67: 130-8. https://doi.org/10.1016/j.archger.2016.07.008

6. Rzewuska M, de Azevedo-Marques JM, Coxon D, Zanetti ML, Zanetti ACG, Franco LJ, Santos JL. Epidemiology of multimorbidity within the brazilian adult general population: evidence from the 2013 National Health survey (PNS 2013). PloS One. 2017;12(2):e0171813. https://doi.org/10.1371/journal.pone.0171813

7. Kingston A, Robinson L, Booth H, Knapp M, Jagger C, MODEM project. Projections of multi-morbidity in the older population in England to 2035: estimates from the Population Ageing and Care Simulation (PACSim) model. Age Ageing. 2018;47(3):374-80. https://doi.org/10.1093/ ageing/afx201

8. Baxter AJ, Patton G, Scott KM, Degenhardt L, Whiteford HA. Global epidemiology of mental disorders: what are we missing? PLoS One. 2013;8(6):e65514. https://doi. org/10.1371/journal.pone.0065514

9. Valiengo LdCL, Stella F, Forlenza OV. Mood disorders in the elderly: prevalence, functional impact, and management challenges. Neuropsychiatr Dis Treat. 2016;12:2105-14. https://doi.org/10.2147/ndt.s94643

10. World Health Organization. Depression and other common mental disorders: global health estimates. geneva: $\mathrm{WHO} ; 2017$.

11. World Health Organization. Global action plan for the prevention and control of noncommunicable diseases 2013-2020. Geneva: WHO; 2013.

12. Quatrin LB, Galli R, Moriguchi EH, Gastal FL, Pattussi MP. Collective efficacy and depressive symptoms in Brazilian elderly. Arch Gerontol Geriatr. 2014;59(3): 624-9. https://doi.org/10.1016/j.archger.2014.08.001

13. Whiteford HA, Ferrari AJ, Degenhardt L, Feigin V, Vos T. The global burden of mental, neurological and substance use disorders: an analysis from the Global Burden of Disease Study 2010. PloS One. 2015;10(2):e0116820. https://doi.org/10.1371/journal.pone.0116820

14. Yu C, Sylvestre JD, Segal M, Looper KJ, Rej S. Predictors of psychiatric re-hospitalization in older adults with severe mental illness. Int J Geriatr Psychiatry. 2015;30(11): 1114-9. https://doi.org/10.1002/gps.4361

15. Wang S, Li B, Ungvari GS, Ng CH, Chiu HF, Kou C, Liu Y, Tao Y, Wu Y, Fu Y, Qi Y, Yu Y, Xiang YT. Poor mental health status and its associations with demographic characteristics and chronic diseases in Chinese elderly. Soc Psychiatry Psychiatr Epidemiol. 2016;51(10):1449-55. https://doi.org/10.1007/s00127-016-1271-y

16. Robards J, Evandrou M, Falkingham J, Vlachantoni A. Marital status, health and mortality. Maturitas. 2012;73(4): 295-9. https://doi.org/10.1016/j.maturitas.2012.08.007 
17. Chen Y, Feeley TH. Social support, social strain, loneliness, and well-being among older adults: An analysis of the Health and Retirement Study. J Soc Pers Relat. 2014;31(2):141-61. https://doi.org/10.1177/ 0265407513488728

18. Stahl ST, Beach SR, Musa D, Schulz R. Living alone and depression: the modifying role of the perceived neighborhood environment. Aging Mental Health. 2017; 21(10):1065-71. https://doi.org/10.1080/13607863.2016. 1191060

19. Munhoz TN, Nunes BP, Wehrmeister FC, Santos IS, Matijasevich A. A nationwide population-based study of depression in Brazil. J Affect Disord. 2016;192:226-33. https://doi.org/10.1016/j.jad.2015.12.038

20. Fujise N, Abe Y, Fukunaga R, Nakagawa Y, Nishi Y, Koyama A, Ikeda M. Comparisons of prevalence and related factors of depression in middle-aged adults between urban and rural populations in Japan. J Affect Disord. 2016;190:772-6. https://doi.org/10.1016/j. jad.2015.11.020

21. Breslau J, Marshall GN, Pincus HA, Brown RA. Are mental disorders more common in urban than rural areas of the United States? J Psychiatr Res. 2014; 56:50-5. https://doi.org/10.1016/j.jpsychires.2014. 05.004

22. Weaver A, Himle JA, Taylor RJ, Matusko NN, Abelson JM. Urban vs rural residence and the prevalence of depression and mood disorder among African American women and non-Hispanic white women. JAMA Psychiatry. 2015;72(6):576-83. https://doi.org/10.1001/ jamapsychiatry.2015.10

23. Sengupta P, Benjamin AI. Prevalence of depression and associated risk factors among the elderly in urban and rural field practice areas of a tertiary care institution in Ludhiana. Indian J Public health. 2015;59(1):3-8. https://doi.org/10.4103/0019-557x.152845

24. Fernández-Niño JA, Bonilla-Tinoco LJ, ManriqueEspinoza BS, Romero-Martínez M, Sosa-Ortiz AL. Work status, retirement, and depression in older adults: An analysis of six countries based on the Study on Global Ageing and Adult Health (SAGE). SSM-Popul Health. 2018;6:1-8. https://doi.org/10.1016/j.ssmph.2018. 07.008

25. Ju YJ, Han KT, Lee HJ, Lee JE, Choi JW, Hyun IS, Park EC. Quality of life and national pension receipt after retirement among older adults. Geriatr Gerontol Int. 2017;17 (8):1205-13. https://doi.org/10.1111/ggi. 12846

26. Hajat C, Kishore SP. The case for a global focus on multiple chronic conditions. BMJ. 2018;3(3):e000874. https://doi.org/10.1136/bmjgh-2018-000874

27. Hoogendijk EO, Rijnhart JJ, Kowal P, Pérez-Zepeda MU, Cesari M, Abizanda P, Flores Ruano T, SchopEtman A, Huisman M, Dent E. Socioeconomic inequalities in frailty among older adults in six lowand middle-income countries: Results from the WHO Study on global AGEing and adult health (SAGE). Maturitas. 2018;115:56-63. https://doi.org/10.1016/j. maturitas.2018.06.011
28. Stubbs B, Vancampfort D, Veronese N, Schofield P, Lin PY, Tseng PT, Solmi M, Thompson T, Carvalho AF, Koyanagi A. Multimorbidity and perceived stress: a population-based cross-sectional study among older adults across six low-and middle-income countries. Maturitas. 2018;107:84-91. https://doi.org/10.1016/j. maturitas.2017.10.007

29. Ryan A, Wallace E, O'Hara P, Smith SM. Multimorbidity and functional decline in community-dwelling adults: a systematic review. Health Qual Life Outcomes. 2015; 13:168. https://doi.org/10.1186/s12955-015-0355-9

30. Adams L, Koop P, Quan H, Norris C. A population-based comparison of the use of acute healthcare services by older adults with and without mental illness diagnoses. J Psychiatr Ment Health Nurs. 2015;22(1):39-46. https://doi.org/10.1111/jpm.12169

31. El-Majzoub S, Behzadi A, Lis E, Moussaoui G, Yu C, Segal M, Looper KJ, Chachamovich E, Rej S. Selfreported psychiatric symptoms associated with anticipated close psychiatry follow-up and/or hospitalization status in geriatric psychiatry patients: a preliminary study. Psychogeriatrics. 2018;18(1):74-6. https://doi.org/10.1111/ psyg.12283

32. Vos T, Allen C, Arora M, Barber RM, Bhutta ZA, Brown A, et al. Global, regional, and national incidence, prevalence, and years lived with disability for 310 diseases and injuries, 1990-2015: a systematic analysis for the Global Burden of Disease Study 2015. Lancet. 2016;388(10053):1545-602.

33. Blazer DG. Depression in late life: review and commentary. J Gerontol A Biol Sci Med Sci. 2003; 58(3):M249-M65.

34. Fiske A, Wetherell JL, Gatz M. Depression in older adults. Annu Rev Clin Psychol. 2009;5:363-89. https://doi. org/10.1146/annurev.clinpsy.032408.153621

35. Ylli A, Miszkurka M, Phillips SP, Guralnik J, Deshpande $\mathrm{N}$, Zunzunegui MV. Clinically relevant depression in old age: An international study with populations from Canada, Latin America and Eastern Europe. Psychiatry Res. 2016;241:236-41. https://doi.org/10.1016/j. psychres.2016.04.096

36. Dols A, Beekman A. Older age bipolar disorder. Psychiatr Clin North Am. 2018;41(1):95-110. https://doi.org/ 10.1016/j.psc.2017.10.008

37. Lima IM, Peckham AD, Johnson SL. Cognitive deficits in bipolar disorders: implications for emotion. Clin Psychol Rev. 2018;59:126-36.

38. Rej S, Quayle W, Forester BP, Dols A, Gatchel J, Chen P, Gough S, Fox R, Sajatovic M, Strejilevich SA, Eyler LT. Measurement tools for assessment of older age bipolar disorder: a systematic review of the recent global literature. Bipolar Disord. 2018;20(4):359-69. https:// doi.org/10.1111/bdi.12566

39. Yu W, Singh SS, Calhoun S, Zhang H, Zhao X, Yang F. Generalized anxiety disorder in urban China: Prevalence, awareness, and disease burden. J Affect Disord. 2018;234: 89-96. https://doi.org/10.1016/j.jad. 2018.02.012 
40. Zhou Y, Cao Z, Yang M, Xi X, Guo Y, Fang M, Lijuan Cheng, Yukai Dua. Comorbid generalized anxiety disorder and its association with quality of life in patients with major depressive disorder. Sci Rep. 2017;7:40511.

\section{AUTHORS:}

LeTíCIA GÜENTER

Médica. Especialista em Psiquiatria. Pontifícia Universidade Católica do Rio Grande do Sul (PUCRS), Porto Alegre, RS, Brasil.

E-mail: leticiaguenter@hotmail.com

Orcid: https://orcid.org/0000-0003-1615-1893

GABRIEL BEHR GOMES JARDIM

Médico. Mestre em Gerontologia Biomédica. Pontifícia Universidade Católica

do Rio Grande do Sul (PUCRS), Porto Alegre, RS, Brasil.

E-mail: gabrielbehr@gmail.com

Orcid: https://orcid.org/0000-0001-7937-8185

FRANCISCO PASCOAL JR

Médico. Mestre em Gerontologia Biomédica. Pontifícia Universidade Católica

do Rio Grande do Sul (PUCRS), Porto Alegre, RS, Brasil.

E-mail: pascoaliunior@uol.com.br

Orcid: https://orcid.org/0000-0001-5827-7270

LUCAS SPANEMBERG

Médico. Doutor em Ciências Médicas: Psiquiatria. Pontifícia Universidade Católica do Rio Grande do Sul (PUCRS), Porto Alegre, RS, Brasil.

E-mail: lucas.spanemberg@pucrs.br

Orcid: https://orcid.org/0000-0001-6789-7420

MARCO ANTÔNIO PACHECO

Médico. Mestre em Medicina e Ciências da Saúde. Pontifícia Universidade Católica do Rio Grande do Sul (PUCRS), Porto Alegre, RS, Brasil.

E-mail: marco.pacheco@pucrs.br

Orcid: https://orcid.org/0000-0003-2565-7781

VANESSA SGNAOLIN

Farmacêutica. Doutora em Gerontologia Biomédica. Pontifícia Universidade Católica do Rio Grande do Sul (PUCRS), Porto Alegre, RS, Brasil.

E-mail: vanessa.sgnaolin@pucrs.br

Orcid: https://orcid.org/0000-0002-9914-7146

Alfredo Cataldo NetO

Médico. Doutor em Medicina e Ciências da Saúde. Pontifícia Universidade Católica do Rio Grande do Sul (PUCRS), Porto Alegre, RS, Brasil.

E-mail: cataldo@pucrs.br

Orcid: https://orcid.org/0000-0002-8082-1866 